\title{
Thioredoxin relieves lipopolysaccharide-induced acute kidney injury in mice by reducing inflammation, oxidative stress and apoptosis
}

\author{
JINGJING WANG ${ }^{1}$, WENJUAN ZHANG ${ }^{1}$ and GUOYUAN LU ${ }^{2}$ \\ ${ }^{1}$ Department of Nephrology, Changzhou Fourth People's Hospital, Changzhou, Jiangsu 213000; \\ ${ }^{2}$ Department of Nephrology, First Affiliated Hospital of Suzhou University, Suzhou, Jiangsu 215000, P.R. China
}

Received May 25, 2020; Accepted March 8, 2021

DOI: $10.3892 /$ etm.2021.10061

\begin{abstract}
Acute kidney injury (AKI) is a serious disease with rapid onset and a high mortality rate. It is therefore particularly important to identify a suitable method for treating AKI. Thioredoxin ( Trx) is a potent anti-inflammatory and anti-oxidant protein that is prevalent in living organisms. The aim of the present study was to facilitate the clinical treatment of AKI via the study of Trx. Lipopolysaccharide (LPS) was used to construct an AKI model in mice and the mice were pre-treated with Trx to examine its effect on AKI. In addition, human renal tubular epithelial HK-2 cells were cultured and stimulated with Trx to examine its effect on inflammation, levels of oxidative stress and apoptosis in the HK-2 cells. The NF- $\kappa \mathrm{B}$ signaling pathway is a classical inflammation-related pathway and the mechanism of Trx was investigated by evaluating the association between $\operatorname{Tr} x$ and the NF- $\mathrm{B}$ signaling pathway. Trx treatment reduced LPS-induced levels of inflammation, oxidative stress and apoptosis in the HK-2 cells. The activity of NF- $\mathrm{BB}$ signaling pathway was increased in LPS-induced HK-2 cells, while Trx treatment effectively reduced NF- $\kappa \mathrm{B}$ signaling pathway activity. In addition, Trx treatment significantly reduced LPS-induced mouse AKI in vivo, which was characterized by a decrease in inflammatory factors in mouse serum, a decrease in AKI-associated molecules in mouse urine and a decrease in oxidative stress levels in mouse kidney tissue samples. Trx treatment reduced inflammation, levels of oxidative stress and apoptosis in HK-2 cells by inhibiting the $\mathrm{NF}-\kappa \mathrm{B}$ signaling pathway, thereby alleviating LPS-induced mouse AKI.
\end{abstract}

Correspondence to: Dr Jingjing Wang, Department of Nephrology, Changzhou Fourth People's Hospital, 68 Honghe Road, Changzhou, Jiangsu 213000, P.R. China

E-mail: wang55848847@163.com

Key words: thioredoxin, acute kidney injury, inflammation, oxidative stress, apoptosis, $\mathrm{NF}-\kappa \mathrm{B}$ signaling pathway

\section{Introduction}

Acute kidney injury (AKI) is caused by a variety of factors and leads to a sharp decline in renal function, which can affect a variety of organs and systems (1). The overall incidence of AKI is estimated to be $0.2-0.3 \%$, the incidence of AKI in hospitalized patients is $10 \%$, the incidence in intensive care units is $22-67 \%$ and the mortality rate is as high as $50-80 \%$ (2). In addition, the overall mortality rate of AKI is $60 \%$ in adults and $57-66 \%$ in children (3). In the USA, 300,000 individuals succumb to AKI each year. Furthermore, AKI seriously affects the quality of life of patients and increases the economic burden on society (4). At present, there is a lack of uniform and effective treatments. Renal replacement therapy (RRT) is currently the only effective supportive treatment. However, under the maintenance of RRT, the mortality rate of AKI remains at 50-60\%. Moreover, with the increasing progress of RRT technology, the aforementioned mortality rate has not changed significantly, and the medical burden generated by AKI has also increased (5). AKI has long been considered a reversible clinical syndrome and previous studies have demonstrated that, although the clinical symptoms of AKI can be restored, it can cause permanent damage to kidney tissue $(5,6)$. Therefore, it is important to establish effective targets for the treatment of AKI.

Renal tubular injury, inflammation and vascular dysfunction are the main pathological features of AKI. Acute tubular necrosis is the most important mechanism of AKI, with damage and death of tubule cells considered to be major markers of AKI severity (7). The recovery and regeneration of tubule cells is considered to be a major marker of renal injury recovery. Apoptosis is an active process and is a type of programmed cell death. However, excessive apoptosis can cause tissue and organ atrophy, resulting in irreversible damage (8).

Thioredoxin (Trx) is a type of protein molecule with a catalytically active dithiol site that is widely present in living organisms. It was first identified as a component of supernatant from human T lymphocyte virus type 1 transformed T cells (9). The Trx system protects proteins and cell membranes from oxidative injury (9). In addition to its antioxidant effects, it regulates cytokine transcription and apoptosis. In reactive oxygen species (ROS)-induced cardiomyocyte injury and 
apoptosis, Trx clears ROS and upregulates DnaJ heat shock protein family (Hsp40) member B5 in signal transduction pathways to enhance mitochondrial function, thereby inhibiting cardiac hypertrophy (10). Numerous studies have found that Trx could protect cells from oxidative and inflammatory injury, while no significant adverse symptoms have been found $(11,12)$.

Therefore, the present study hypothesized that the anti-inflammatory and anti-oxidative features of Trx may be useful in the treatment of AKI. To verify this, C57/BL6 mice were used to construct an AKI model and were administered Trx. In addition, the underlying mechanism of Trx was investigated using HK-2 cells.

\section{Materials and methods}

Animals and grouping. The present study was approved by the Animal Ethics Committee of Changzhou Fourth People's Hospital Animal Center. A total of 80 C57/BL6 male mice (age, 8 weeks; weight, $20 \pm 2 \mathrm{~g}$ ) were obtained from Changzhou Fourth People's Hospital Animal Center. All mice were housed in a specific pathogen free laboratory animal room at a temperature of $22-24^{\circ} \mathrm{C}$ and a relative humidity of $50-60 \%$. Mice received specific-pathogen-free grade food and drinking water, ad libitum. An artificial $12 \mathrm{~h}$ light/dark cycle with suitable ventilation was maintained in the animal room. Mice were divided into three groups as follows: Control group, AKI group and AKI + Trx (Abcam) group. Mice in the AKI group and the AKI + Trx group were used to construct the AKI model. Mice in the AKI + Trx group were administered a daily subcutaneous injection of recombinant mouse $\operatorname{Trx}(50 \mathrm{mg} / \mathrm{kg})$ one week prior to the construction of the AKI model (13). Mice in the control group were routinely housed in squirrel cages and were injected with the same quantity of saline.

Surgical procedure for mouse AKI model construction. Lipopolysaccharide (LPS) was used to induces sepsis in mice (14). The mice were anesthetized with pentobarbital sodium at a dose of $40 \mathrm{mg} / \mathrm{kg}$ intraperitoneally. The mice were then administered $10 \mathrm{mg} / \mathrm{kg}$ LPS (Sigma-Aldrich; Merck KGaA) intraperitoneally using a microsyringe. After the injection, the syringe was gently rotated $90^{\circ}$ clockwise and slowly extracted. After the modeling was completed, all of the mice were returned to the squirrel cage for rearing. After $24 \mathrm{~h}$, mouse urine was obtained for analysis and the mice were sacrificed by cervical dislocation (14).

Enzyme linked immunosorbent assay (ELISA). The expression levels of relevant indicators in the serum, urine and cellular supernatant of mice were evaluated by ELISA. ELISA kits [Jianglai Biotechnology (Jianglaibio) Co., Ltd. and Hangzhou Multisciences (Lianke) Biotech, Co., Ltd.] were used to detect the expression of neutrophil gelatinase-associated lipocalin (NGAL), kidney injury molecule 1 (KIM-1), netrin-1 and liver-type fatty acid binding protein (L-FABP) in mouse urine. In addition, the eyeballs of the mice were removed for blood collection and the serum was obtained by centrifugation $\left(3,000 \mathrm{xg}\right.$ for $5 \mathrm{~min}$ at $\left.4^{\circ} \mathrm{C}\right)$. The ELISA kits were used to detect the expression of serum creatinine [Scr; cat. no. JL20633; Jianglai Biotechnology (Jianglaibio) Co., Ltd.], blood urea nitrogen [BUN; cat. no. JL20491; Jianglai Biotechnology (Jianglaibio) Co., Ltd.], IL-1 $\beta$ [cat. no. 70-EK201B/3-96; Hangzhou Multisciences (Lianke) Biotech, Co., Ltd.] and TNF- $\alpha$ [cat. no. 70-EK282/3-96; Hangzhou Multisciences (Lianke) Biotech, Co., Ltd.] in the serum of the mice. The expression of IL-1 $\beta$ [cat. no. 70-EK101BHS-96; Hangzhou Multisciences (Lianke) Biotech, Co., Ltd.], IL-6 [cat. no. 70-EK106/2-96; Hangzhou Multisciences (Lianke) Biotech, Co., Ltd.] and TNF- $\alpha$ [cat. no. 70-EK182HS-96; Hangzhou Multisciences (Lianke) Biotech, Co., Ltd.] in the cellular supernatant was also detected by ELISA.

Malondialdehyde (MDA) and superoxide dismutase (SOD) activity assay. The mouse kidneys were collected and stored at $-80^{\circ} \mathrm{C}$. The kidney tissue was ground into powder at $4^{\circ} \mathrm{C}$, then dissolved in phosphate buffered saline (PBS). The level of oxidative stress in mouse kidneys was determined by measuring the levels of MDA and SOD in solution. MDA and SOD kits were obtained from Hangzhou Multisciences (Lianke) Biotech, Co., Ltd. and were used to detect the levels of MDA and SOD in the kidneys of mice in accordance with the manufacturer's instructions.

Hematoxylin and eosin $(H \& E)$ staining. After the AKI models were constructed, the mice were sacrificed and their kidneys were harvested. After stripping the renal capsule of the mouse kidney, the kidneys were fixed with $4 \%$ paraformaldehyde at room temperature. After $48 \mathrm{~h}$, the kidneys were washed with PBS and embedded in paraffin by dehydration. A microtome was used to slice the paraffin sections at $5 \mu \mathrm{m}$. After the paraffin sections were dried, an H\&E kit (Beyotime Institute of Biotechnology) was used to perform $\mathrm{H} \& \mathrm{E}$ staining. The kidney injury was evaluated according to the method of Paller et al (15). An optical microscope (Leica Microsystems, Inc.) was used to record the results. Ten diseased tubules were randomly selected from the field of view at high magnification (x200) and the kidney tissue was scored by two members (JW and WZ). Marked dilatation of renal tubules or flattening of cells was scored as 1 point; damage to the brush border was scored as 1 point; cell shedding was scored as 2 points; the renal tubular cells were scored as 2 points; irregularly shaped or fragmented cells are scored as 1 point.

Cell culture and treatment. HK-2 cells (Procell Life Science \& Technology Co., Ltd.) were cultured in Dulbecco's modified Eagle's medium (HyClone) containing 10\% fetal bovine serum (HyClone) and placed in an incubator at $37^{\circ} \mathrm{C}$ and $5 \% \mathrm{CO}_{2}$ until further processing. After the cell growth density reached $50-60 \%$, the cells were stimulated with LPS or Trx. LPS was used to stimulate HK-2 cells to induce AKI at the cellular level.

Western blot analysis. When the HK-2 cell growth density reached $\geq 90 \%$, the cells were passaged into 6-well plates and stimulated with Trx or LPS. After the treatment was complete, the cells were lysed with protein lysate and the protein concentration was detected using a bicinchoninic acid (BCA) kit (Beyotime Institute of Biotechnology). The Murine kidney samples were also lysed using RIPA lysis 
Table I. Reverse transcription-quantitative PCR primers.

Sequence (5'-3')

Primer

Human
Sense
Anti-sense

\begin{tabular}{lll}
\hline SOD1 & GGTGAACCAGTTGTGTTGTC & CCGTCCTTTCCAGCAGTC \\
SOD2 & CAGACCTGCCTTACGACTATGG & CTCGGTGGCGTTGAGATTGT \\
GPX1 & ATCATATGTGTGCTGCTCGGCTAGC & TACTCGAGGGCACAGCTGGGCCCTTGAG \\
GPX3 & AGAGCCGGGGACAAGAGAA & ATTTGCAGCATACTGCTTGA \\
Catalase & CGTACTGATCTATGCGCTCTACA & GCTACTGAGCCACACTGAGCACGA \\
Caspase-3 & CAGAATCATAAGCCCCTGGA & TCTGCGAGTCAGGCATTTG \\
Caspase-9 & TTCTTGAGCAACACCCTC & CGCATACACTGTCTACCT \\
Bax & CAGTTGAAGTTGCCATCAGC & CAGTTGAAGTACCATCAGC \\
Bcl-2 & GACTGAGTACCTGAACCGGCATC & CTGAGCAGCGTCTTCAGAGACA \\
p65 & ACTGCCGGGATGGCTACTAT & TCTGGATTCGCTGGCTAATGG \\
IKK $\alpha$ & GTCAGGACCGTGTTCTCAAGG & GCTTCTTTGATGTTACTGAGGGC \\
IкB $\alpha$ & GGATCTAGCAGCTACGTACG & TTAGGACCTGACGTAACACG \\
GAPDH & ACAACTTTGGTATCGTGGAAGG & GCCATCACGCCACAGTTC \\
Mouse & & \\
p65 & CCAGGGTGTGTCCATGTCT & GTGTGGGAGCTGGGTCA \\
IKK $\alpha$ & GTGGCCCTCAGTAACATCA & AAGAGAGCGGGCAGAAC \\
I $\alpha \alpha$ & CGATGAATGGTGCGACAG & GCCCAGGAGGAAATCCA \\
GAPDH & GCAGTGGCAAAGTGGAG & GCCGTGAGTGGAGTCATAC \\
\hline
\end{tabular}

SOD, superoxide dismutase; GP, glutathione peroxidases

buffer (Beyotime Institute of Biotechnology) to produce the protein lysate, the concentration of which was determined using a BCA kit (Beyotime Institute of Biotechnology). Protein $(20 \mu \mathrm{g})$ was added to each well of the electrophoresis gel and the different components of the protein were separated by $10 \%$ sodium dodecyl sulphate-polyacrylamide gel electrophoresis. The protein was then transferred to a polyvinylidene fluoride (PVDF) membrane (EMD Millipore), which was blocked with $5 \%$ skimmed milk-PBST for $1 \mathrm{~h}$ at room temperature. The PVDF membrane was incubated at $4^{\circ} \mathrm{C}$ overnight with the following primary antibodies: IL-1 $\beta$ (1:3,000; cat. no. 31202S; CST Biological Reagents Co., Ltd.), IL-6 (1:3,000; cat. no. ab9324; Abcam), IL-8 (1:3,000; cat. no. ab154390; Abcam), TNF- $\alpha$ (1:3,000; cat. no. ab183218; Abcam), caspase-3 (1:3,000; cat. no. ab184787; Abcam), caspase-9 (1:2,000; cat. no. ab202068; Abcam), p65 (1:5,000; cat. no. ab32536; Abcam), IKK $\alpha$ (1:4,000; cat. no. ab32041; Abcam), IкB $\alpha$ (1:4,000; cat. no. ab32518; Abcam) and $\beta$-actin $(1: 5,000$; cat. no. ab8226; Abcam). The next day the PVDF membrane was washed with PBST and incubated at room temperature for $2 \mathrm{~h}$ using goat anti-rabbit secondary antibody (1:5,000; cat. no. ab6721; Abcam). Finally, an electrochemiluminescence kit (Sigma-Aldrich; Merck KGaA) was used to detect the protein quantity. ImageJ Software 1.52 (National Institutes of Health) was used for gray value analysis.

$R N A$ isolation and reverse transcription-quantitative $P C R$ (RT-qPCR). TRIzol ${ }^{\circledR}$ (Invitrogen; Thermo Fisher Scientific,
Inc.) was used to extract total RNA from the HK-2 cells and the RNA concentration was detected using a spectrophotometer $(260 \mathrm{~nm})$. The reverse transcription reaction system was prepared on ice to avoid the degradation of RNA. After reverse transcription was completed, an SYBR Green kit (Invitrogen; Thermo Fisher Scientific, Inc.) was used to amplify cDNA with different primers (Table I). The expression of endogenous GAPDH served as the control and the $2^{-\Delta \Delta \mathrm{Cq}}$ method was used to statistically analyze the relative expression levels of the target genes (16).

Cell counting kit-8 (CCK-8) assay. HK-2 cells were cultured in 96 -well plates. After the cell growth density reached $60 \%$, $0,10,30,50$ and $100 \mathrm{ng} / \mathrm{ml}$ of Trx was administered to stimulate the HK-2 cells. CCK-8 reagent (10 $\mu$; ; Dojindo Molecular Technologies, Inc.) was added to each well of the 96-well plate and the cells were returned to the incubator for an additional $2 \mathrm{~h}$. A microplate reader was used to measure the absorbance of each well.

Immunocytofluorescence (IF) staining. HK-2 cells were cultured in 24-well plates. When the cell growth density reached $60 \%$, the cells were treated as aforementioned. The 12 -well plate was removed and the medium was discarded. After washing with PBS, cells were fixed with $4 \%$ paraformaldehyde for $15 \mathrm{~min}$ at room temperature. The cells were soaked for 20 min using $0.2 \%$ Triton-PBS. After washing cells with PBS, the cells were incubated with $10 \%$ goat serum-PBS for $30 \mathrm{~min}$ at room temperature. The goat serum was discarded and 
the cells were incubated with the following primary antibody dilution: IL-6 (1:300; cat. no. ab9324; Abcam), IL-8 (1:200; cat. no. ab154390; Abcam), SOD1 (1:500; cat. no. ab51254; Abcam), SOD2 (1:800; cat. no. ab110300; Abcam), Bax (1:400; cat. no. ab53154; Abcam), Bcl-2, (1:400; cat. no. ab218123; Abcam) and p65 (1:200; cat. no. ab32536; Abcam) at $4^{\circ} \mathrm{C}$ overnight. After washing the cells with PBS the next day, the cells were incubated for $1 \mathrm{~h}$ using fluorescent secondary antibody, goat anti-rabbit-FITC (1:500; cat. no. ab150077; Abcam). The cells were incubated for 15 min with 4',6-diamidino-2-phenylindole at room temperature. After washing the cells with PBS, the outcome of staining was observed and recorded using a fluorescence microscope.

Flow cytometry. ROS levels and apoptosis rates were detected in HK-2 cells by flow cytometry. After cell growth density reached $60 \%$, the cells were treated as aforementioned. We then used the DCFH-DA kit (Invitrogen; Thermo Fisher Scientific, Inc.) and the Annexin V-FITC/PI kit (Invitrogen; Thermo Fisher Scientific, Inc.) to determine ROS levels and apoptosis rates in HK-2 cells according to the manufacturer's instructions. Flow cytometry (Thermo Fisher Scientific, Inc.; cat. no. A28997) was used to detect apoptosis with Attune NxT Software 3.2.1 (Thermo Fisher Scientific, Inc.).

Statistical analysis. All experiments were repeated more than three times. SPSS V21.0 (IBM Corp.) was used for statistical analysis and GraphPad Prism 7.0 (GraphPad Software, Inc.) was used to construct and present the data. Comparisons between multiple groups were performed using One-way ANOVA followed by the least significant difference post hoc test. Kruskal-Wallis test followed by Dunn's test was were used for the histological scoring. $\mathrm{P}<0.05$ was considered to indicate a statistically significant difference.

\section{Results}

Trx reduces LPS-induced inflammation levels in HK-2 cells. HK-2 cells were cultured and stimulated with Trx. A CCK-8 assay was used to determine the optimal concentration of Trx for HK-2 cells. Results indicated that $50 \mathrm{ng} / \mathrm{ml}$ Trx achieved optimal cell viability (Fig. 1A). The HK-2 cells were then stimulated with different concentrations of LPS and the results demonstrated that $1 \mu \mathrm{g} / \mathrm{ml}$ LPS significantly reduced the activity of HK-2 cells (Fig. 1B). When stimulating HK-2 cells with both LPS $(1 \mu \mathrm{g} / \mathrm{ml})$ and $\operatorname{Trx}, 50 \mathrm{ng} / \mathrm{ml} \operatorname{Trx}$ significantly increased the activity of HK-2 cells (Fig. 1C). The results of ELISA showed that inflammatory factors (IL-1 $\beta$, IL-6 and TNF- $\alpha$ ) were significantly increased in HK-2 cells of the LPS group and Trx could reduce the expression of inflammatory factors (Fig. 1D-F). The results of IF staining also indicated that Trx reduced IL-6 (Fig. 1G) and IL-8 (Fig. 1H). The results of western blot analysis were similar to those of ELISA and IF staining (Fig. 1I and J).

Trx reduces $L P S$-induced oxidative stress levels in $\mathrm{HK}-2$ cells. Oxidative stress is a notable feature in the development of AKI. SOD is an important antioxidant enzyme in the body. The results of IF staining showed that the expression of SOD1 and SOD2 in the LPS group was significantly lower than that in the control group, indicating that LPS reduced the anti-oxidant capacity of HK-2 cells. Following stimulation of HK-2 cells with Trx, the anti-oxidant capacity of HK-2 cells increased significantly (Fig. 2A and B). In addition, the results of RT-qPCR showed that Trx increases the expression of SOD1, SOD2, glutathione peroxidases (GP)X1, GPX3 and catalase (CAT; Fig. 2C-G). GPX1/3 is an important peroxidase enzyme that is widely expressed in various organisms and CAT is a key enzyme that catalyzes the breakdown of hydrogen peroxide. The results of flow cytometry indicated that Trx reduces ROS levels in HK-2 cells (Fig. 2H). These results demonstrate that Trx reduces oxidative stress levels in HK-2 cells.

Trx reduces LPS-induced apoptosis in HK-2 cells. The pathological process of AKI is accompanied by the apoptosis of a large number of renal tubular epithelial cells, therefore, the effect of Trx on the apoptosis of HK-2 cells was detected. The results of IF staining indicated that Trx significantly reduced the expression of Bax in HK-2 cells and increased the expression of Bcl-2 (Fig. 3A and B). The results of flow cytometry also indicated that Trx reduced the apoptosis rate in HK-2 cells (Fig. 3C). Western blot analysis (Fig. 3D and E) and RT-qPCR (Fig. 3F-I) showed that the expression of Bax, caspase-3 and caspase-9 in LPS-induced HK-2 cells was significantly increased while the expression of Bcl-2 was decreased. Treatment with Trx attenuated the effects of LPS. The mRNA expression levels of Bax and $\mathrm{Bcl}-2$ were compared and $\mathrm{Trx}$ reduced the $\mathrm{Bax} / \mathrm{Bcl}-2$ ratio (Fig. 3J).

Trx reduces activity of the $N F-\kappa B$ signaling pathway. $\mathrm{NF}-\kappa \mathrm{B}$ is widespread in glomerular epithelial cells, renal tubular epithelial cells and mesangial cells, and is involved in the production and regulation of cytokines, such as TNF- $\alpha$, IL-1 $\beta$ and interferon in the pathogenesis of glomerulonephritis, renal toxicity damage and renal disease (17). To evaluate the underlying mechanism by which Trx attenuated AKI, the activity of the NF- $\kappa \mathrm{B}$ signaling pathway was examined in $\mathrm{HK}-2$ cells. P65, IKK $\alpha$ and $\mathrm{I} \kappa \mathrm{B} \alpha$ are the key signaling molecules in the $\mathrm{NF}-\kappa \mathrm{B}$ signaling pathway. The results of IF staining indicated that $\operatorname{Trx}$ reduces the expression of $\mathrm{p} 65$ (Fig. 4A). The western blot analysis (Fig. 4B) demonstrated that the expression of $\mathrm{p} 65$ and IKK $\alpha$ in the LPS group was significantly higher than that in the control group, while the expression of IкB $\alpha$ was decreased. Treatment with Trx attenuated the effect of LPS. Similar results have also been shown in vivo (Fig. 4C). Quantitative analysis of protein expression was performed (Fig. 4D and E). The results of RT-qPCR indicated that Trx reduced the mRNA expression of p65 and $\mathrm{IKK} \alpha$, and promoted the mRNA expression of $\mathrm{I} \kappa \mathrm{B} \alpha$ in vitro and in vivo (Fig. $4 \mathrm{~F}$ and $\mathrm{G}$ ).

Exogenous Trx attenuates LPS-induced mouse AKI. Trx was subcutaneously injected into mice to verify the effect of Trx on AKI in mice. The results of H\&E staining revealed that some tubular epithelial cells in the AKI group disappeared and inflammatory cells infiltrated the stroma. The tubular epithelial cells in the Trx-treated mice were almost intact, and the renal histopathology was significantly improved 

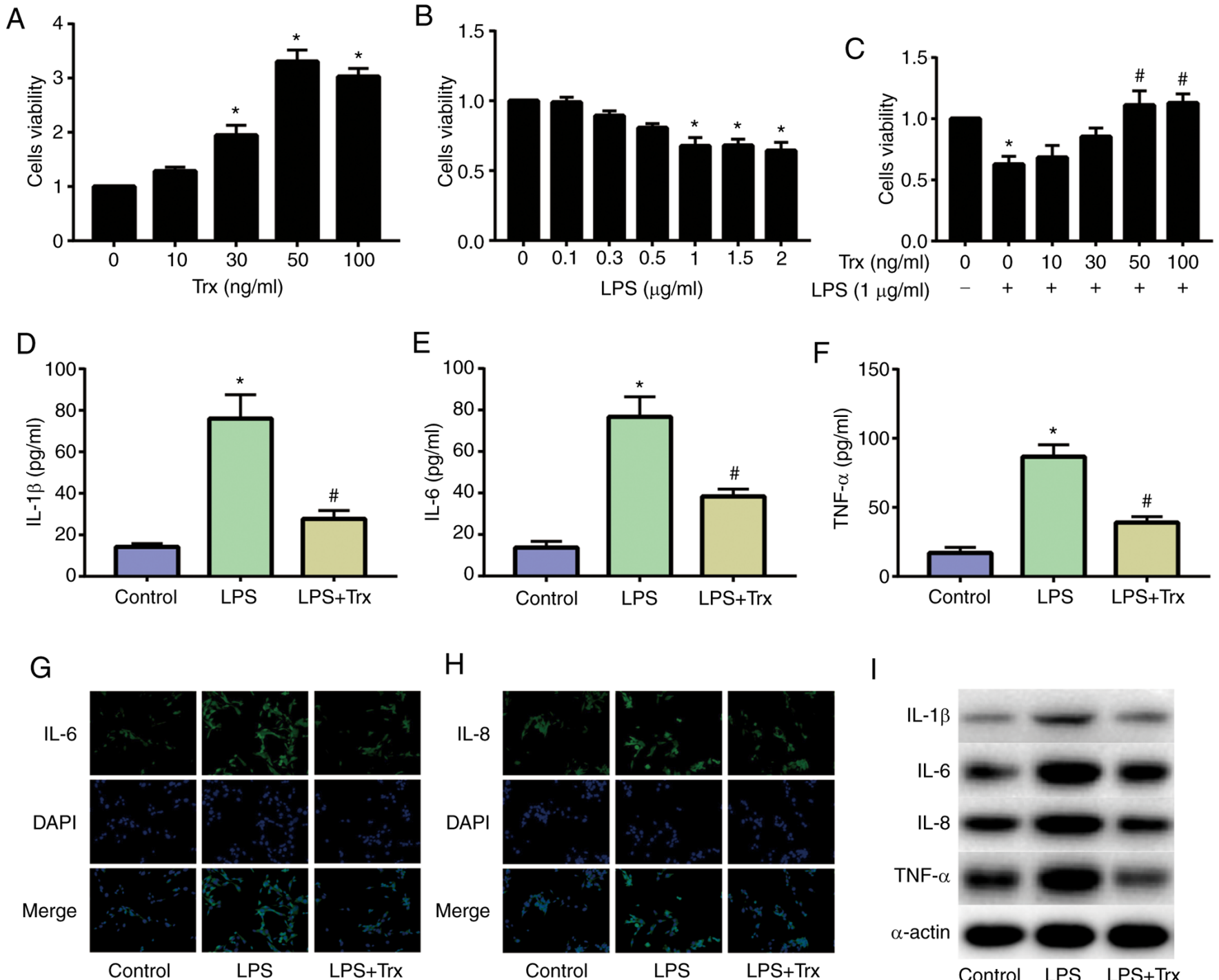

$\mathrm{H}$
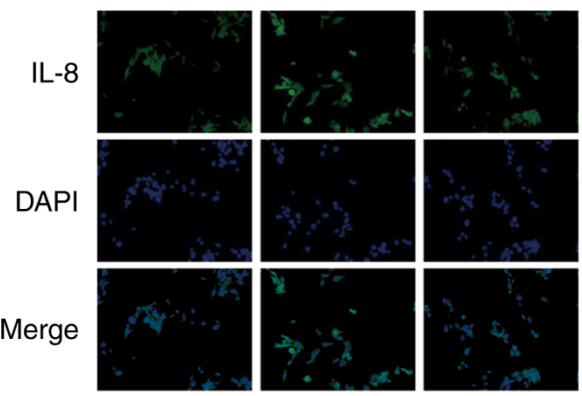

I
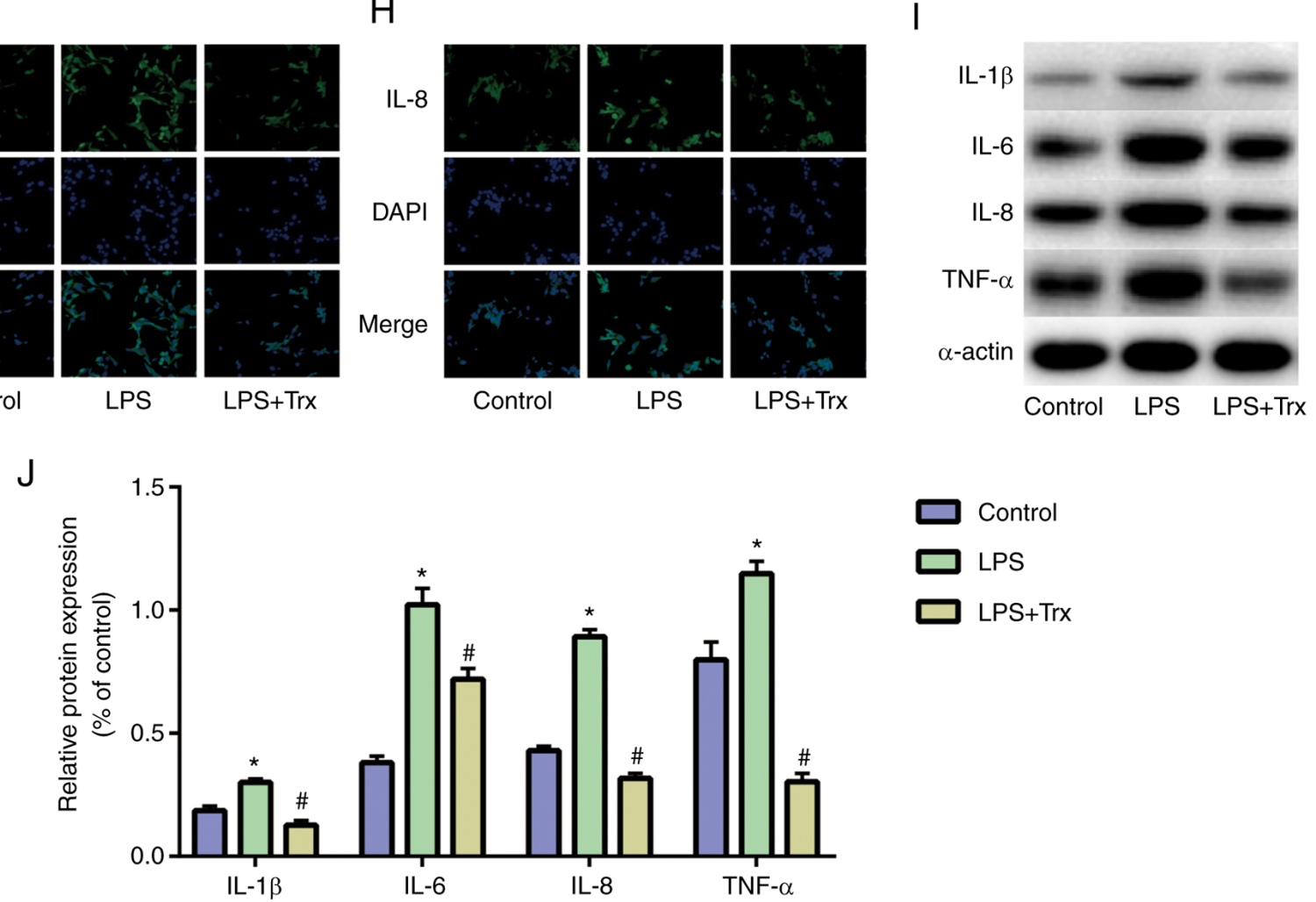

Figure 1. Trx reduces LPS-induced inflammation levels in HK-2 cells. (A) CCK-8 assay of (A) Trx, (B) LPS and (C) Trx + LPS. "P $<0.05$ vs. the 0 group; ${ }^{*} \mathrm{P}<0.05$ vs. the $0 \mathrm{ng} / \mathrm{ml} \mathrm{Trx}+1 \mu \mathrm{g} / \mathrm{ml}$ LPS group. (D-F) ELISA of IL-1 $\beta$, IL-6 and TNF- $\alpha$. (G and H) Immunocytofluorescence staining of IL-6 and IL-8 (magnification, $\mathrm{x} 400$ ). (I and J) Western blot and gray value analysis of IL-1 $\beta$, IL-6, IL-8 and TNF- $\alpha .{ }^{*} \mathrm{P}<0.05$ vs. the control group; ${ }^{*} \mathrm{P}<0.05$ vs. the LPS group. Data are presented as the mean \pm SD. Trx, thioredoxin; LPS, lipopolysaccharide; CCK-8, Cell Counting Kit-8.

when compared with that of the AKI group (Fig. 5A and B). MDA and SOD are important indicators of oxidative stress. The expression levels of MDA and SOD in mouse kidney tissue samples were detected by ELISA. It was found that Trx effectively increased the expression of SOD and decreased the expression of MDA, indicating that Trx reduced the level of oxidative stress in mouse kidney tissues (Fig. 5C and D). In addition, the expression of AKI biomarkers, NGAL, KIM-1, netrin-1 and L-FABP, in the urine of mice in the AKI group was significantly higher than that in the control group, while the expression of AKI biomarkers in the urine of mice in the AKI + Trx group was significantly reduced (Fig. 5E-H). 
A

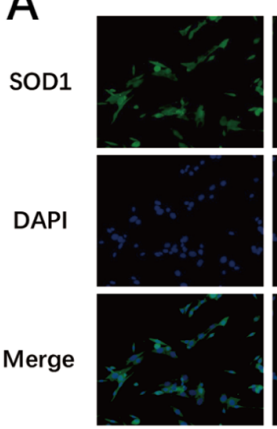

control

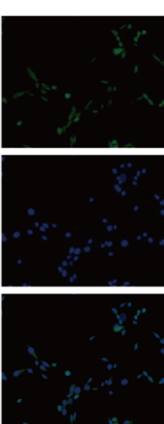

LPS

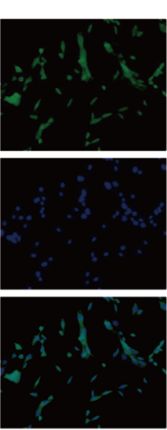

LPS+Trx

C

D

B

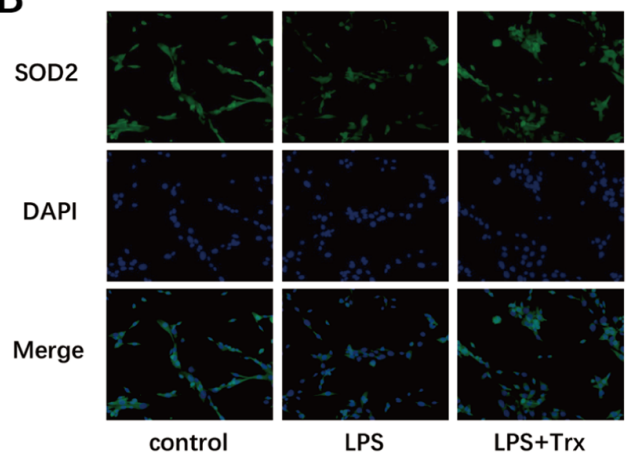

$E$
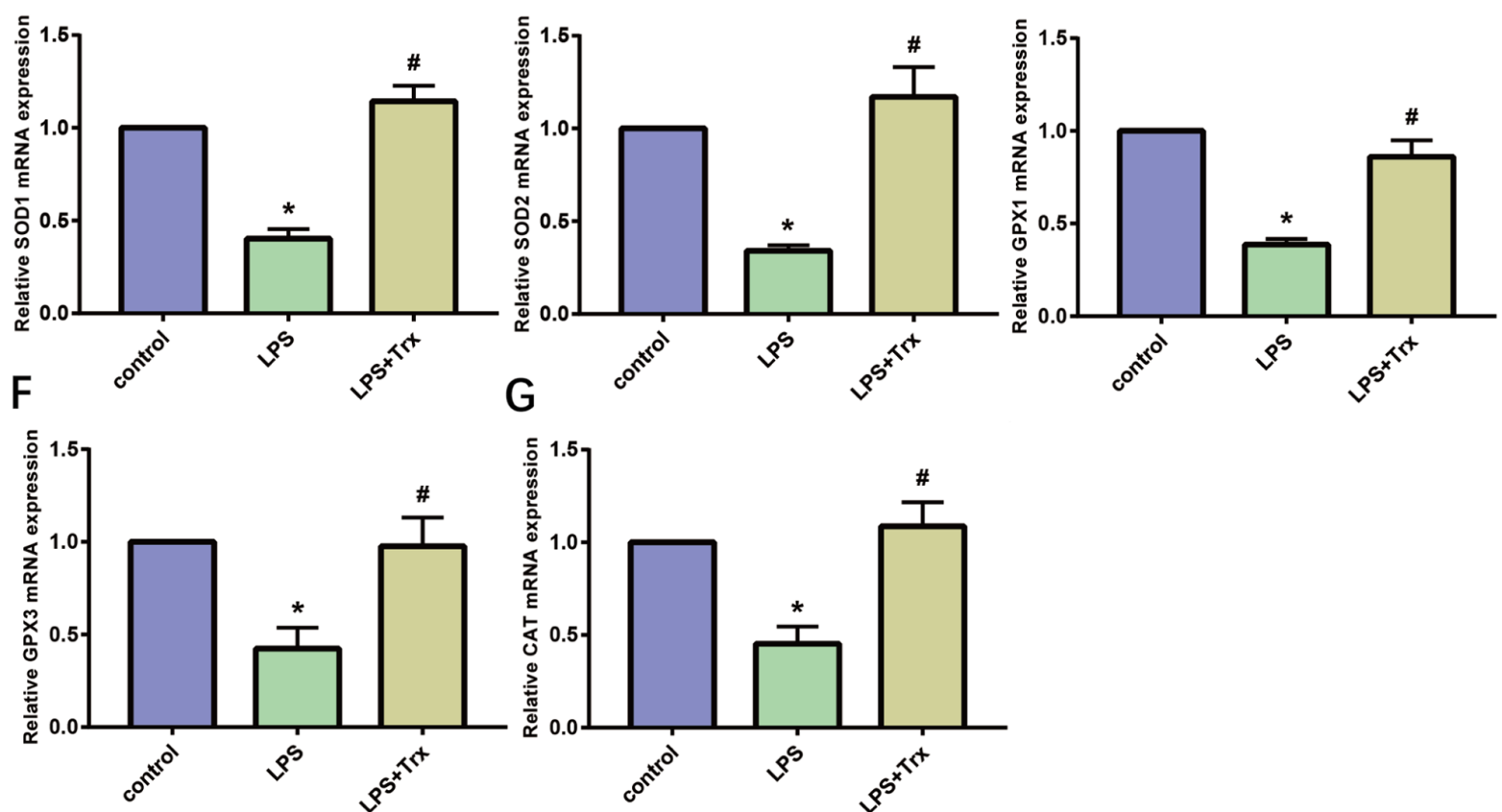

H
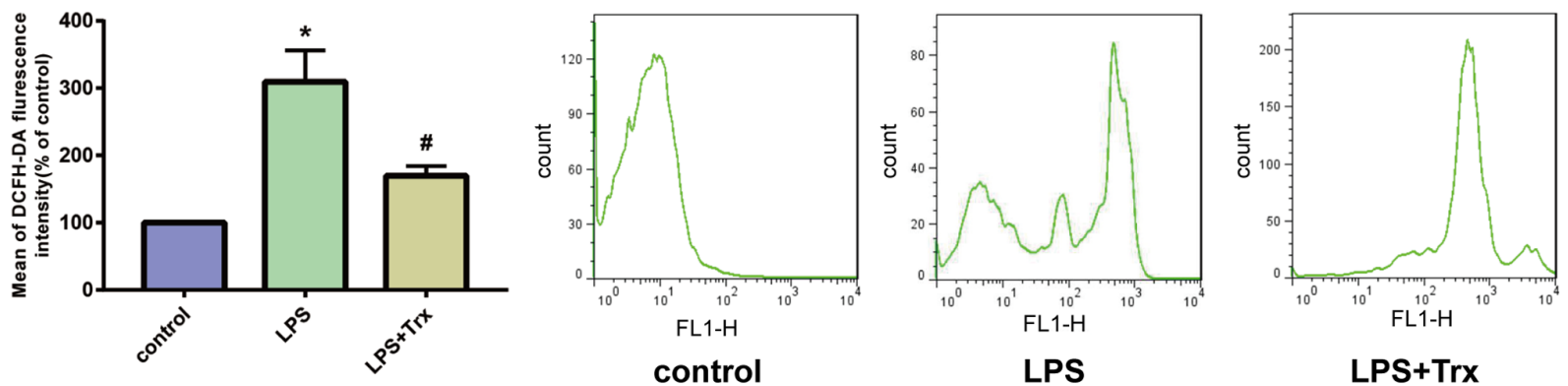

Figure 2. Trx reduces LPS-induced oxidative stress levels in HK-2 cells. (A and B) Immunocytofluorescence staining of SOD1 and SOD2 (magnification, $\mathrm{x} 400$ ). (C-G) Reverse transcription-quantitative PCR of SOD1, SOD2, GPX1, GPX3 and CAT. (H) ROS level in HK-2 cells. *P<0.05 vs. the control group; ${ }^{\#} \mathrm{P}<0.05$ vs. the LPS group. Data are presented as the mean \pm SD. Trx, thioredoxin; LPS, lipopolysaccharide; SOD, superoxide dismutase; GP, glutathione peroxidase; CAT, catalase; ROS, reactive oxygen species.

The expression levels of Scr, BUN, IL-1 $\beta$ and TNF- $\alpha$ in the serum of LPS-induced mice were higher than that of the control group. Treatment with Trx decreased their expression (Fig. 5I-L).

\section{Discussion}

After AKI, tubular epithelial cells swell, necrosis and shedding are observed, and the kidneys exhibit localized ischemia, hypoxia and oxidative stress cascades, accompanied by inflammatory cell infiltration. These complex pathophysiological processes initiate the self-repairing function of the kidneys, but the kidneys have limited ability to regenerate compared to organs with abundant blood supply and powerful functions, such as the heart and liver. When injury is severely beyond the kidney load, the kidney cannot complete self-repair and the remaining renal tubular epithelial cells lose cell polarity, showing continuous and uncontrolled proliferation of abnormalities (18). 
A

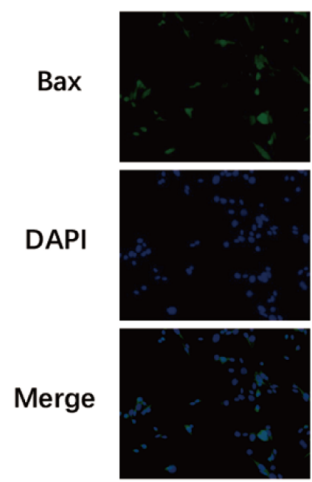

control
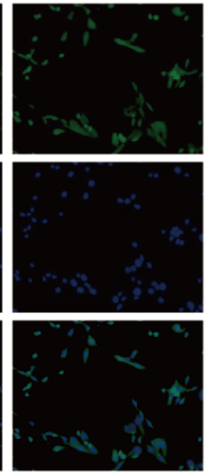

LPS
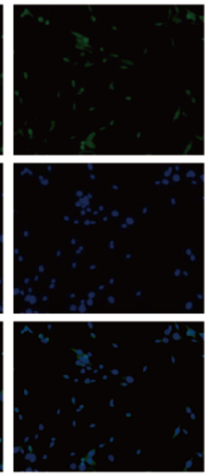

LPS+Trx

B

Bcl-2
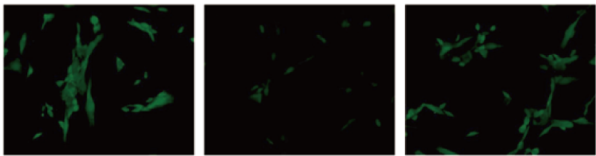

DAPI

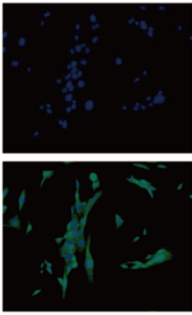

control

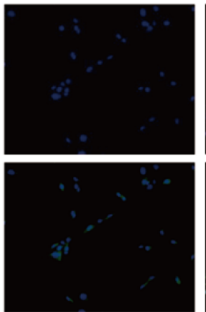

LPS

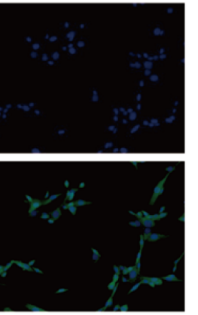

LPS + Trx
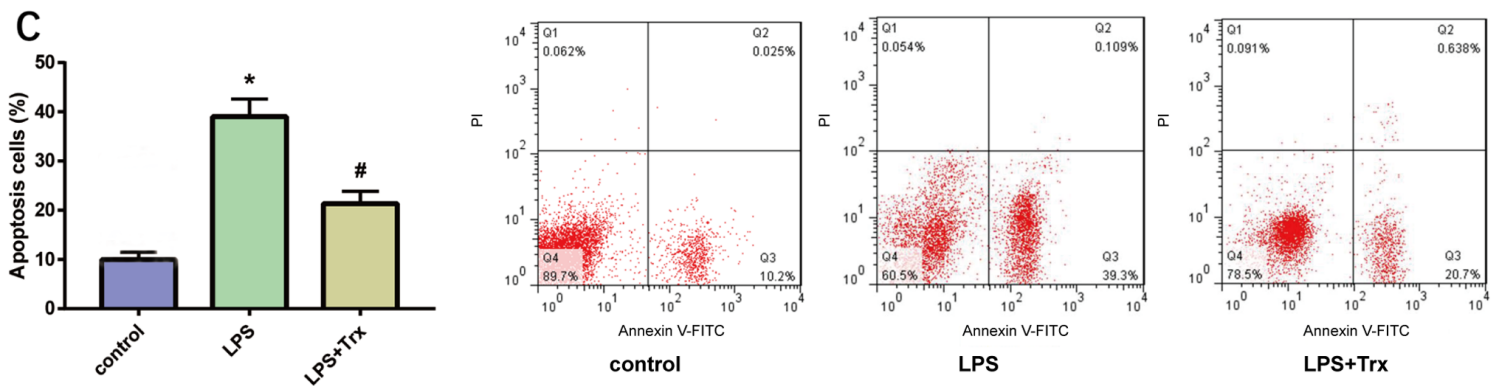

D

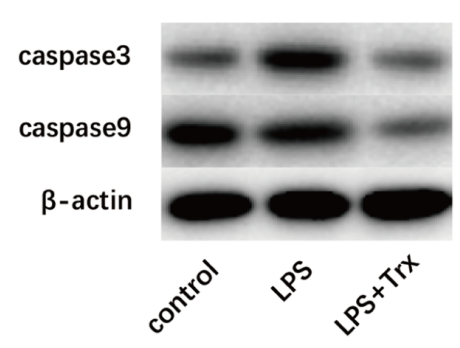

E
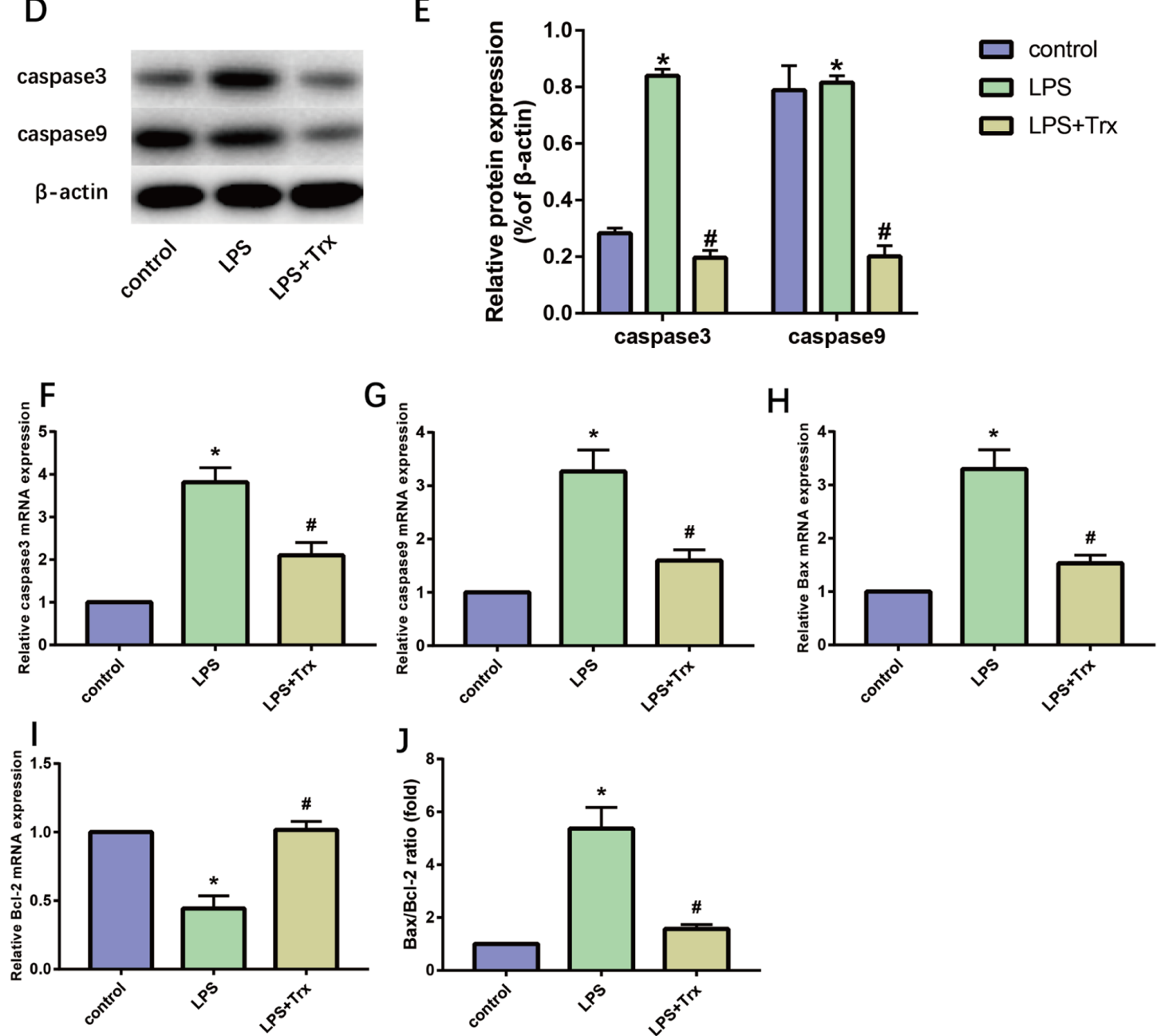

Figure 3. Trx reduces LPS-induced apoptosis in HK-2 cells. (A and B) Immunocytofluorescence staining of Bax and Bcl-2 (magnification, $\mathrm{x} 400$ ). (C) Percentage of apoptotic cells in HK-2 cells. (D and E) Western blot and gray value analysis of caspase-3 and caspase-9. (F-I) Reverse transcription-quantitative PCR of caspase-3, caspase-9, Bax and Bcl-2. (J) Bax/Bcl-2 ratio of RNA expression. ${ }^{*} \mathrm{P}<0.05$ vs. the control group. "P<0.05 vs. the LPS group. Data are presented as the mean \pm SD. Trx, thioredoxin; LPS, lipopolysaccharide. 
A

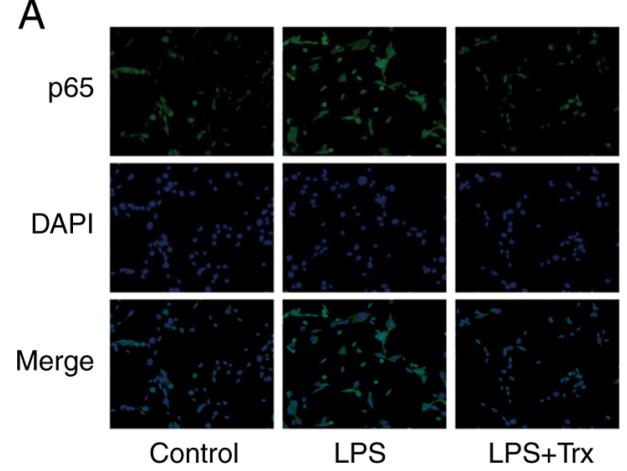

B

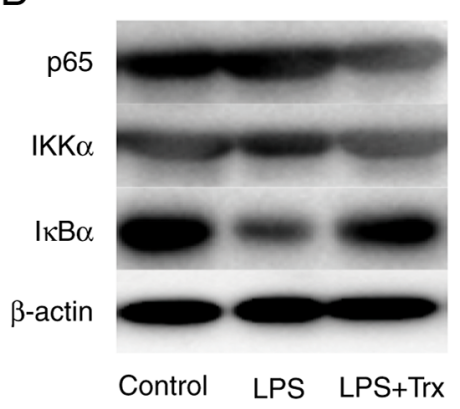

C

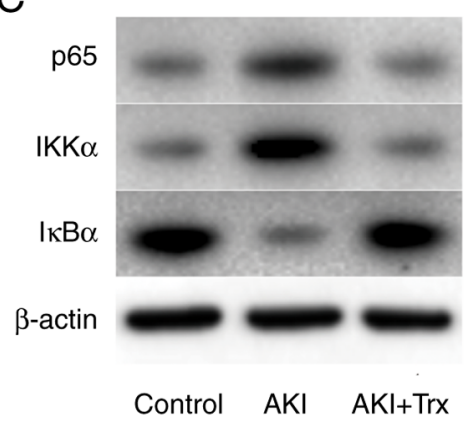

D

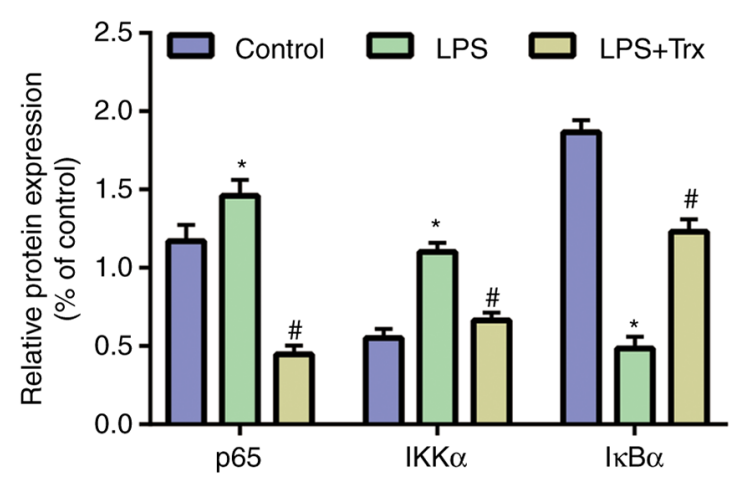

$\mathrm{F}$

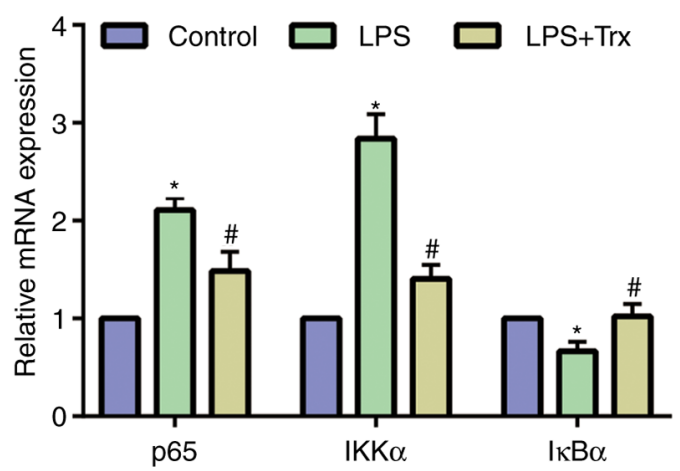

E

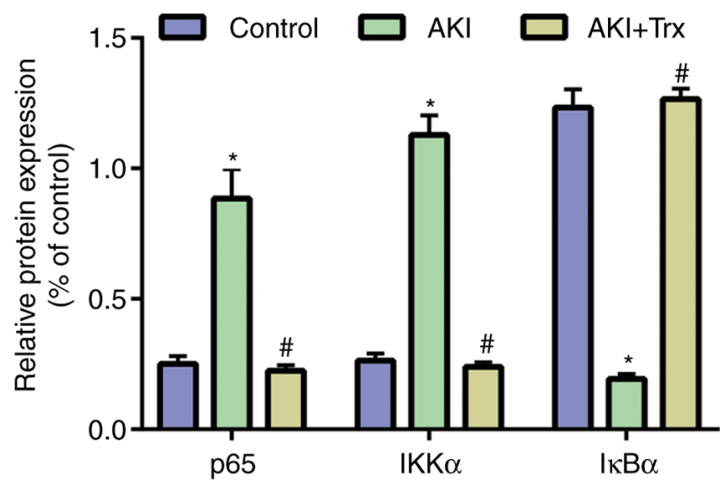

G

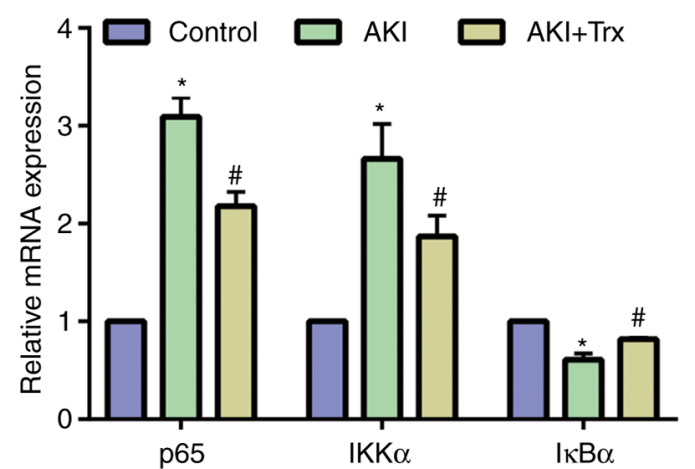

Figure 4. Trx reduces NF- $\mathrm{B}$ signaling pathway activity. (A) Immunocytofluorescence staining of p65 (magnification, $\mathrm{x} 400$ ). (B-E) Western blot and gray value analysis of $\mathrm{p} 65, \mathrm{IKK} \alpha$ and $\mathrm{I} \kappa \mathrm{B} \alpha$ in vitro and in vivo. ( $\mathrm{F}$ and $\mathrm{G})$ Reverse transcription-quantitative $\mathrm{PCR}$ of $\mathrm{p} 65$, IKK $\alpha$ and I $\kappa \mathrm{B} \alpha$ in vitro and in vivo. ${ }^{*} \mathrm{P}<0.05$ vs. the control group; ${ }^{\#} \mathrm{P}<0.05$ vs. the LPS group or the AKI group. Data are presented as the mean \pm SD. Trx, thioredoxin; LPS, lipopolysaccharide; AKI, acute kidney injury.

These paracrine products of renal tubular epithelial cells also interfere with the interaction between normal renal tubular epithelial cells, perivascular capillary endothelial cells and pericytes, resulting in epithelial to mesenchymal transformation, endothelial to mesenchymal transformation and pericytes to myofibroblast transformation (19). Myofibroblasts continue to proliferate and activate, secreting a large quantity of collagen fibers deposited in the renal parenchyma, gradually developing into renal interstitial fibrosis and progressing to end-stage renal disease (19). Renal tubular epithelial cell injury is the initiating factor for the progression of AKI to chronic kidney disease (20). Therefore, interventions for renal tubular epithelial cells will effectively promote kidney repair and regeneration.

Numerous clinical studies have shown that elevated levels of Trx in plasma or serum can be used as clinical parameters for inflammatory diseases $(21,22)$. Trx can be secreted into saliva and respond to the severity of the disease through the level of salivary Trx. Trx also regulates macrophage migration inhibitory factor (MIF) to exert anti-inflammatory effects (23). MIF has attracted widespread attention as a deteriorating factor in inflammatory diseases (23). A gene fusion protein, human serum albumin (HSA)-Trx (consisting of HSA and Trx) has a long half-life and good biological activity. HSA-Trx has a certain effect in the treatment of oxidative stress-related diseases. HSA-Trx also inhibits the production of inflammatory cytokines by inhibiting MIF to exert an anti-inflammatory effect. In a previous study, transgenic mice overexpressing Trx suppressed allergies and inflammation in an allergic contact dermatitis (ACD) model. Following sensitization with 1-fluoro-2,4-dinitrofluorobenzene, no difference in the antigen-specific proliferation of lymph node cells was observed in the Trx transgenic mice and control mice (24). Therefore, overexpression of Trx did not affect the primary 
A

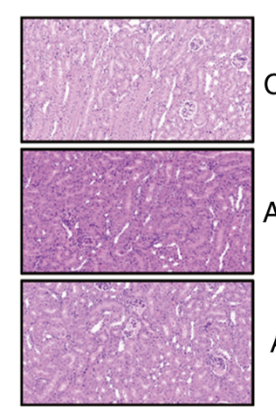

B

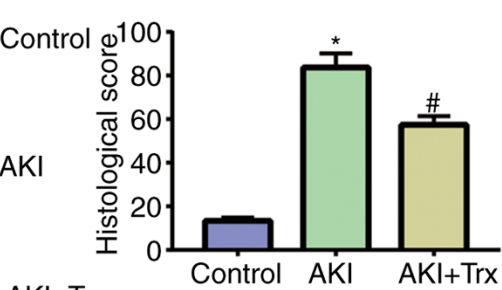

$A K I+T r x$

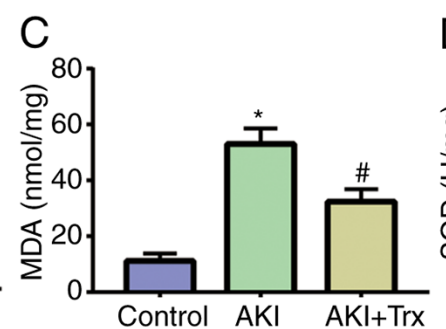

D

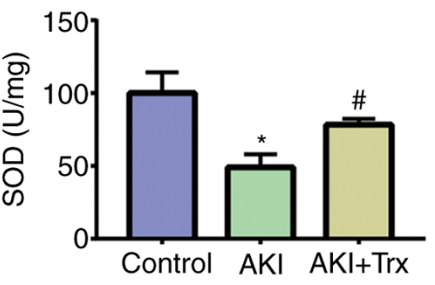

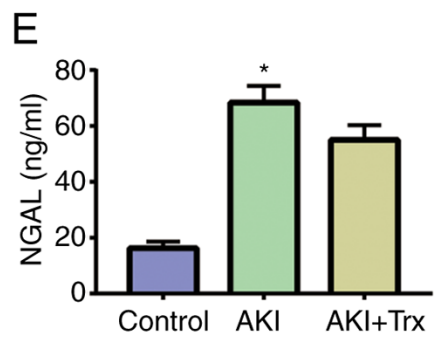

F

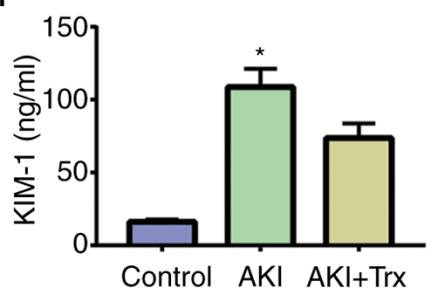

$J$

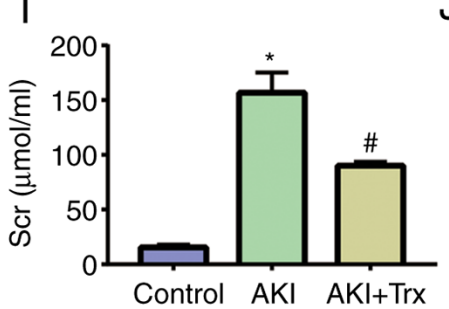

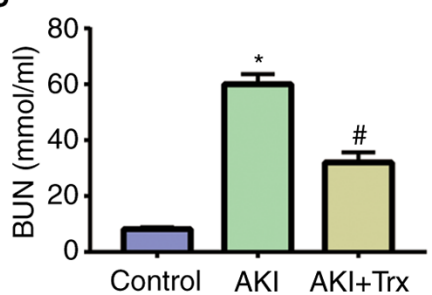

G

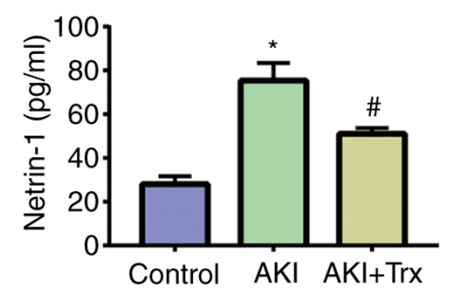

K

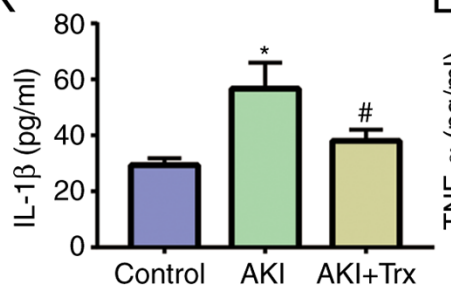

$\mathrm{H}$

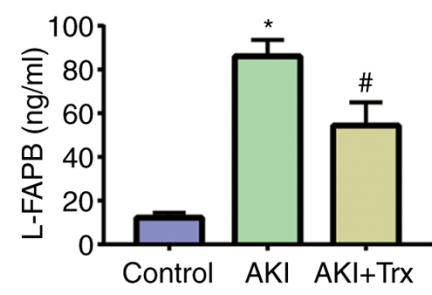

L

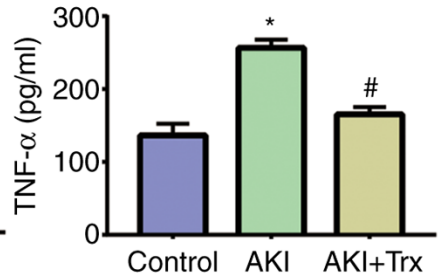

Figure 5. Exogenous Trx attenuates LPS-induced mouse AKI. (A and B) H\&E staining and histological scoring of mouse kidney samples (magnification, x100). (C) MDA and (D) SOD activity in mouse kidney samples. (E-H) ELISA of NGAL, KIM-1, netrin-1 and L-FABP. (I-L) ELISA of Scr, BUN, IL-1 $\beta$ and TNF- $\alpha$. ${ }^{*} \mathrm{P}<0.05$ vs. the control group; ${ }^{*} \mathrm{P}<0.05$ vs. the LPS group. Data are presented as the mean \pm SD. Trx, thioredoxin; LPS, lipopolysaccharide; AKI, acute kidney injury; MDA, malondialdehyde; SOD, superoxide dismutase; NGAL, neutrophil gelatinase-associated lipocalin; KIM-1, kidney injury molecule 1; L-FABP, liver-type fatty acid binding protein; Scr, serum creatinine; BUN, blood urea nitrogen.

immune response during $\mathrm{ACD}$, and neutrophil infiltration was reduced. In addition, skin inflammation was inhibited. These findings indicated that Trx could reduce inflammation during the induction phase of ACD and its anti-inflammatory mechanism was different to other anti-inflammatory agents. The protective effect of exogenous Trx was also confirmed in a mouse model of irritant contact dermatitis (ICD) (25). Recombinant human Trx can inhibit the production and release of pro-inflammatory mediators at the site of inflammation and inhibit ICD (25). Furthermore, IL-1 $\beta$ produced by inflammatory cells stimulates renal epithelial cells to produce downstream inflammatory cytokines, TNF- $\alpha$ and IL-6 (26). Similarly, the inhibitory effect of Trx on inflammatory cytokines (IL-1 $\beta$ and TNF- $\alpha$ ) was observed in the serum of AKI mice in the present study.

MDA is a product of lipid peroxidation and its content reflects the level of oxygen free radicals and the speed of lipid oxidation in the body $(27,28)$. MDA in the serum of AKI mice was also found to be inhibited by Trx in the present study. SOD is a free radical scavenging factor in vivo and its activity reflects the ability of cells to scavenge free radicals and resist lipid peroxidation. SOD plays a key role in preventing the formation of peroxides, anti-oxidative stress injury and anti-renal tubular injury (29). The present study also identified the augmenting effect of Trx on SOD in AKI mice. The ratio of anti-apoptotic molecule $\mathrm{Bcl} 2$ to pro-apoptotic molecule Bax reflects the level of apoptosis. An increase in Bax and a decrease in Bcl2 lead to increases in the caspase family, thereby contributing to cell apoptosis (30). In the present study, Trx treatment significantly reduced LPS-induced AKI in mice, predominantly manifesting as decreased levels of inflammation and oxidative stress. Trx not only reduced Scr and BUN in the mouse serum, but also decreased the expression of AKI-related molecules in urine samples. In addition, Trx reduced inflammatory factors, oxidative stress levels and apoptosis levels in HK-2 cells, demonstrating significant anti-inflammatory, anti-oxidative and anti-apoptosis effects, which are beneficial for relieving AKI.

The NF- $\kappa \mathrm{B}$ signaling pathway is a typical inflammatory signaling pathway, which is largely based on the pivotal position of $N F-\kappa B$ in the expression of inflammatory genes (22). $\mathrm{NF}-\kappa \mathrm{B}$ could increase the expression of cytokines, chemokines, adhesion factors, matrix metalloproteinases, cyclooxygenase 2 and nitric oxide synthase, and NF- $\mathrm{NB}$ itself is activated and sustained in numerous inflammatory diseases (31). Inflammatory bowel disease, glomerulonephritis and septic shock have been identified as inflammatory diseases closely

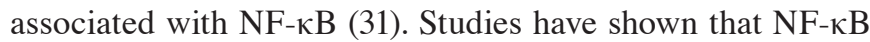
can be activated by endotoxin to enter the nucleus and regulate the gene expression of various inflammatory mediators, particularly the increase of TNF- $\alpha$ and IL-1 $\beta$, which further acts on macrophages and other inflammatory mediators and 
cytokines $(32,33)$. These cytokines further activate NF- $\mathrm{kB}$, thus forming a positive feedback cascade amplification effect, producing an excessive inflammatory response and causing extensive tissue and cell injury (33). Inflammation associated cytokine storms during the course of sepsis tends to damage the mitochondria in cells. Damaged mitochondria produce a large quantity of ROS and antioxidant enzymes cannot completely eliminate the excess ROS. Inflammation associated cytokine storms and oxidative stress lead to various types of cell damage, including apoptosis and even death (34).

The NF- $\mathrm{KB}$ signaling pathway and its downstream inflammatory cascade play an important role in LPS-induced AKI. In previous studies, the expression of pro-inflammatory cytokines, toll-like receptor 4 and phosphorylated p65 in renal tissue were all increased in AKI animal models induced by different stimuli. Blocking or inhibiting the expression of these molecules with drugs prevented the subsequent inflammatory response and promoted kidney tissue repair (35-37). Therefore, it is critical for the treatment of these diseases to inhibit the NF- $\kappa B$ signaling pathway. In the present study, LPS-induced $N F-\kappa B$ signaling pathway activity in HK-2 cells was significantly increased, indicating that the NF- $\mathrm{KB}$ signaling pathway was involved in the development of LPS-induced AKI. The activity of the NF- $\mathrm{kB}$ signaling pathway was decreased in Trx-stimulated HK-2 cells, indicating that Trx can inhibit the NF- $\mathrm{KB}$ signaling pathway in HK-2 cells. Therefore, the role of Trx in improving AKI may be related to inhibition of the NF- $\mathrm{KB}$ signaling pathway. However, the present study did not conduct Trx dose-dependency experiments, and further investigations are required to establish the optimal treatment modality and mechanism of action of Trx.

The present study identified that Trx reduced LPS-induced inflammation, oxidative stress and apoptosis levels in HK-2 cells. In addition, Trx reduced the activity of the NF- $\mathrm{KB}$ signaling pathway in HK-2 cells. The protective effect of Trx on HK-2 cells was also verified in vivo. Trx effectively alleviated LPS-induced mouse AKI, reduced inflammatory factors in mouse serum and reduced MDA levels in kidney tissue samples. To conclude, Trx treatment reduced inflammation, levels of oxidative stress and apoptosis in HK-2 cells by inhibiting the NF- $\mathrm{KB}$ signaling pathway, thereby alleviating LPS-induced mouse AKI.

\section{Acknowledgements}

Not applicable.

\section{Funding}

No funding was received.

\section{Availability of data and materials}

All data generated or analyzed during this study are included in this published article.

\section{Authors' contributions}

JW designed the study and performed the experiments. WZ and GL established the animal models. WZ and GL collected the data and JW, WZ and GL analyzed the data. JW prepared the manuscript. All authors read and approved the final manuscript. JW and WZ confirm the authenticity of all the raw data.

\section{Ethics approval and consent to participate}

This study was approved by the Animal Ethics Committee of Changzhou Fourth People's Hospital Animal Center.

\section{Patient consent for publication}

Not applicable.

\section{Competing interests}

The authors declare that they have no competing interests.

\section{References}

1. Wang YM, Han RL, Song SG, Yuan XP and Ren XS: Inhibition of PARP overactivation protects acute kidney injury of septic shock. Eur Rev Med Pharmacol Sci 22: 6049-6056, 2018.

2. Pakula AM and Skinner RA: Acute kidney injury in the critically Ill patient: A current review of the literature. J Intensive Care Med 31: 319-324, 2016.

3. Wald R, Kitchlu A, Harel Z and Silver SA: Care of the acute kidney injury survivor. Nephron 137: 306-309, 2017.

4. Skube SJ, Katz SA, Chipman JG and Tignanelli CJ: Acute kidney injury and sepsis. Surg Infect (Larchmt) 19: 216-224, 2018.

5. Rahman M, Shad F and Smith MC: Acute kidney injury: A guide to diagnosis and management. Am Fam Physician 86: 631-639, 2012.

6. Rydén L, Hertzberg D, Sartipy U and Holzmann M: Acute kidney injury is a common and serious condition. The clinical significance is great and probably underestimated. Lakartidningen 113: DXD3, 2016 (In Swedish).

7. Kampaktsis PN, Feldman DN and Charitakis K: Strategies to avoid TAVI-related acute kidney injury. Curr Pharm Des 22: 1950-1958, 2016.

8. Zhang J, Li X, Han X, Liu R and Fang J: Targeting the thioredoxin system for cancer therapy. Trends Pharmacol Sci 38: 794-808, 2017.

9. Léveillard T and Aït-Ali N: Cell signaling with extracellular thioredoxin and thioredoxin-like proteins: Insight into their mechanisms of action. Oxid Med Cell Longev 2017: 8475125, 2017.

10. Mattoo RU, Farina Henriquez Cuendet A, Subanna S, Finka A, Priya S, Sharma SK and Goloubinoff P: Synergism between a foldase and an unfoldase: Reciprocal dependence between the thioredoxin-like activity of DnaJ and the polypeptide-unfolding activity of DnaK. Front Mol Biosci 1: 7, 2014.

11. Jia J, Zeng X, Xu G and Wang Z: The potential roles of redox enzymes in Alzheimer's disease: Focus on thioredoxin. ASN Neuro 13: 1759091421994351, 2021.

12. Yin Z, Ren H, Liu L, Chen W, Gan C, Jiao H and Fan J: Thioredoxin protects skin flaps from ischemia-reperfusion Injury: A novel prognostic and therapeutic target. Plast Reconstr Surg 137: 511-521, 2016

13. Ueda S, Nakamura T, Yamada A, Teratani A, Matsui N, Furukawa S, Hoshino Y, Narita M, Yodoi J and Nakamura H: Recombinant human thioredoxin suppresses lipopolysaccharide-induced bronchoalveolar neutrophil infiltration in rat. Life Sci 79: 1170-1177, 2006.

14. Shen J, Cui ZK, Yao F, Li K, Zhang Y, Chen Z, Zhou Y, Xu S, Zhang Y, Jiang W, et al: TSC1 deletion in fibroblasts alleviates lipopolysaccharide-induced acute kidney injury. Clin Sci (Lond) 132: 2087-2101, 2018.

15. Paller MS, Hoidal JR and Ferris TF: Oxygen free radicals in ischemic acute renal failure in the rat. Clin Invest 74: 1156-1164, 1984.

16. Livak KJ and Schmittgen TD: Analysis of relative gene expression data using real-time quantitative PCR and the 2(-Delta Delta C(T)) method. Methods 25: 402-408, 2001. 
17. Sanz AB, Sanchez-Niño MD, Ramos AM, Moreno JA Santamaria B, Ruiz-Ortega M, Egido J and Ortiz A: NF-kappaB in renal inflammation. J Am Soc Nephrol 21: 1254-1262, 2010.

18. Peerapornratana S, Manrique-Caballero CL, Gómez $\mathrm{H}$ and Kellum JA: Acute kidney injury from sepsis: Current concepts, epidemiology, pathophysiology, prevention and treatment. Kidney Int 96: 1083-1099, 2019.

19. Fragasso T, Ricci Z and Goldstein SL: Pediatric acute kidney injury. Contrib Nephrol 193: 113-126, 2018.

20. Abdelraheem MB: Acute kidney injury in low-and middle-income countries: Investigations, management and prevention. Paediatr Int Child Health 37: 269-272, 2017.

21. Chen G, An N, Ye W, Huang S, Chen Y, Hu Z, Shen E, Zhu J, Gong W, Tong G, et al: bFGF alleviates diabetes-associated endothelial impairment by downregulating inflammation via S-nitrosylation pathway. Redox Biol 41: 101904, 2021.

22. Zhou J and Chng WJ: Roles of thioredoxin binding protein (TXNIP) in oxidative stress, apoptosis and cancer. Mitochondrion 13: 163-169, 2013.

23. Benhar M: Application of a thioredoxin-trapping mutant for analysis of the cellular nitrosoproteome. Methods Enzymol 585: 285-294, 2017.

24. Ouyang Y, Peng Y, Li J, Holmgren A and Lu J: Modulation of thiol-dependent redox system by metal ions via thioredoxin and glutaredoxin systems. Metallomics 10: 218-228, 2018.

25. Mata-Pérez C and Spoel SH: Thioredoxin-mediated redox signalling in plant immunity. Plant Sci 279: 27-33, 2019.

26. Lv Y, Bing Q, Lv Z, Xue J, Li S, Han B, Yang Q, Wang X and Zhang Z: Imidacloprid-induced liver fibrosis in quails via activation of the TGF- $\beta 1 /$ Smad pathway. Sci Total Environ 705 $135915,2020$.

27. Liu B, Yu H, Baiyun R, Lu J, Li S, Bing Q, Zhang X and Zhang Z: Protective effects of dietary luteolin against mercuric chloride-induced lung injury in mice: Involvement of AKT/Nrf2 and NF- $\kappa$ B pathways. Food Chem Toxicol 113: 296-302, 2018.

28. Yang Q, Han B, Xue J, Lv Y, Li S, Liu Y, Wu P, Wang X and Zhang Z: Hexavalent chromium induces mitochondrial dynamics disorder in rat liver by inhibiting AMPK/PGC-1 $\alpha$ signaling pathway. Environ Pollut 265: 114855, 2020.
29. Lv Y, Jiang H, Li S, Han B, Liu Y, Yang D, Li J, Yang Q, Wu P and Zhang Z: Sulforaphane prevents chromium-induced lung injury in rats via activation of the Akt/GSK-3 $\beta /$ Fyn pathway. Environ Pollut 259: 113812, 2020.

30. Li S, Baiyun R, Lv Z, Li J, Han D, Zhao W, Yu L, Deng N, Liu Z and Zhang Z: Exploring the kidney hazard of exposure to mercuric chloride in mice: Disorder of mitochondrial dynamics induces oxidative stress and results in apoptosis. Chemosphere 234: 822-829, 2019.

31. Chen J and Chen LF: Methods to detect NF- $\mathrm{BB}$ acetylation and methylation. Methods Mol Biol 1280: 395-409, 2015.

32. Neale PA, Leusch FDL and Escher BI: What is driving the NF- $\kappa B$ response in environmental water extracts? Chemosphere 210: 645-652, 2018

33. Lawrence T: The nuclear factor NF-kappaB pathway in inflammation. Cold Spring Harb Perspect Biol 1: a001651, 2009.

34. Turkmen K: Inflammation, oxidative stress, apoptosis, and autophagy in diabetes mellitus and diabetic kidney disease: The four horsemen of the apocalypse. Int Urol Nephrol 49: 837-844, 2017.

35. Lee JW, Kim SC, Ko YS, Lee HY, Cho E, Kim MG, Jo SK, Cho WY and Kim HK: Renoprotective effect of paricalcitol via a modulation of the TLR4-NF- $\kappa \mathrm{B}$ pathway in ischemia/reperfusion-induced acute kidney injury. Biochem Biophys Res Commun 444: 121-127, 2014.

36. Nair AR, Masson GS, Ebenezer PJ, Del Piero F and Francis J: Role of TLR4 in lipopolysaccharide-induced acute kidney injury: Protection by blueberry. Free Radic Biol Med 71: 16-25, 2014.

37. Korrapati MC, Shaner BE and Schnellmann RG: Recovery from glycerol-induced acute kidney injury is accelerated by suramin. J Pharmacol Exp Ther 341: 126-136, 2012.

This work is licensed under a Creative Commons

Attribution-NonCommercial-NoDerivatives 4.0

International (CC BY-NC-ND 4.0) License. 